\title{
Pulmonary arteriovenous fistula in a patient with renal cell carcinoma
}

\author{
S. Kopetz*, C. Jimenez ${ }^{\#}$, S-M. Tu and P. Sharma"
}

ABSTRACT: Arteriovenous (AV) fistulas are common in renal cell carcinoma. These are usually small and asymptomatic. Case reports have previously described symptomatic fistulas in primary renal cell tumour or, less commonly, bone metastases.

The current study describes a patient with lung metastases from renal cell carcinoma who developed a symptomatic pulmonary AV fistula. The patient presented with debilitating progressive shortness of breath, which resolved after coiling of the pulmonary AV fistula. Supporting radiographs include a unique ventilation-perfusion finding of perfusion tracer in the kidney that is diagnostic of a pulmonary shunt.

This is the first report of a pulmonary fistula from renal cell carcinoma. This rare complication of renal cell carcinoma reflects its diverse clinical presentations and unique tumour biology.

KEYWORDS: Arteriovenous fistula, pulmonary fistula, renal cell carcinoma, shunt physiology

\section{CASE REPORT}

A 73-yr-old female with metastatic renal cell carcinoma (RCC) presented with progressive shortness of breath. She was initially diagnosed with RCC in February 1992, and underwent a left radical nephrectomy. Shortly after resection, she was found to have bibasilar lung nodules and mediastinal lymphadenopathy, which was subsequently confirmed to be metastatic RCC. She was treated with interferon (IFN)- $\alpha$ in combination with a continuous infusion of 5-fluorouracil followed by floxuridine and had a partial response. She was maintained on floxuridine and IFN- $\alpha$ with intermittent treatment breaks until March 2000, when floxuridine was removed from the market. She subsequently stopped taking IFN- $\alpha$ due to worsening depression.

In May 2003, the patient was noted to have progressive disease in the lungs, and therapy was initiated with capecitabine and gemcitabine, at which time she started to complain of increasing shortness of breath. Previously, she had been an active member in her community; however, she now found it difficult, because of her dyspnoea, to sing in her church choir or volunteer at a local nursing home. Due to the progression of her lung lesions, she was restarted on low-dose IFN- $\alpha$ in February 2004, and her disease stabilised. In November 2004, she had an episode of small-volume haemoptysis, and, at that time, her oxygen saturation was $85 \%$ on room air. The patient was started on home oxygen at $2 \mathrm{~L} \cdot \mathrm{min}^{-1}$. Despite oxygen supplementation, the patient continued to complain of increasing shortness of breath. In January 2005, she reported that she was experiencing shortness of breath even at minimal exertion, such as taking a shower or getting dressed.

In February 2005, she was admitted to the hospital for further evaluation of her shortness of breath and was found to have an oxygen saturation of $84 \%$ on room air and $90 \%$ on $4 \mathrm{~L}$ of oxygen by nasal cannula. A $100 \%$ nonrebreather mask increased her oxygen saturation to $94 \%$. Her physical examination showed clear lungs, borderline tachycardia and cyanosis, but she had no digital clubbing. A chest radiograph showed stable, low-volume pulmonary metastases with no airspace disease or cardiomegaly. A computed tomographic (CT) scan of the lung showed no change from the study performed 2 months previously (fig. 1). A CT angiogram and ventilation-perfusion scan showed no evidence of acute pulmonary embolism. An arteriovenous (AV) shunt was suspected clinically because of the refractory hypoxia. Ventilation-perfusion imaging demonstrated uptake of technetium-99m macroaggregate albumin in the kidneys (fig. 2).
AFFILIATIONS

*Division of Cancer Medicine Depts of "Pulmonary Medicine, and "Genitourinary Medical Oncology,

The University of Texas M.D. Anderson Cancer Center, Houston, TX, USA.

\section{CORRESPONDENCE}

P. Sharma

Dept of Genitourinary Medical Oncology

Unit 1374

The University of Texas M.D.

Anderson Cancer Center

1515 Holcombe Blvd.

Houston

TX 77030

USA

Fax: 17137451625

E-mail:PadSharma@

mdanderson.org

Received:

June 132006

Accepted:

December 062006

STATEMENT OF INTEREST

None declared.

European Respiratory Journal Print ISSN 0903-1936 Online ISSN 1399-3003 


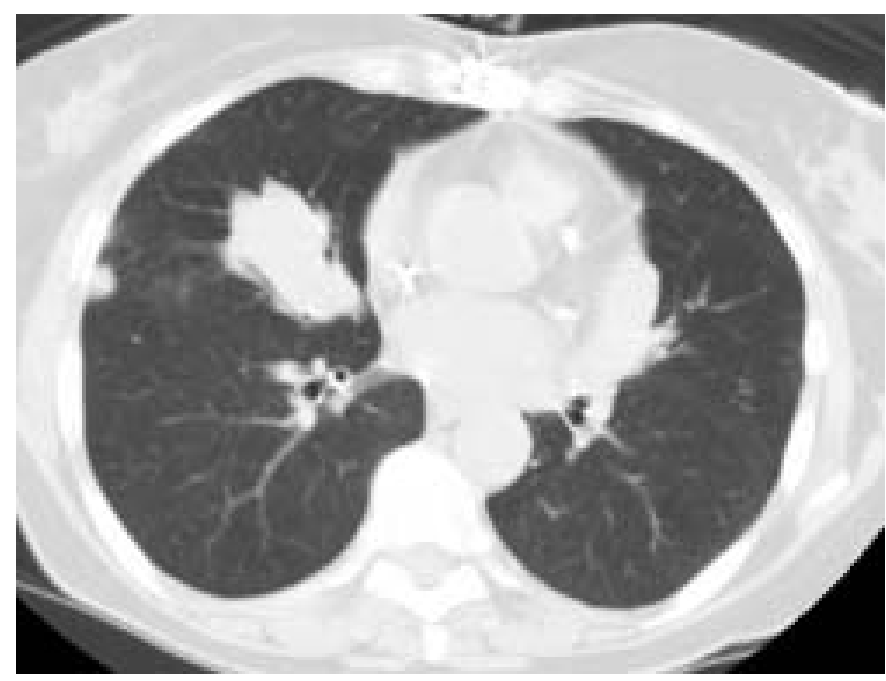

FIGURE 1. Computed tomographic scan of the lung demonstrating metastatic foci of renal cell carcinoma.

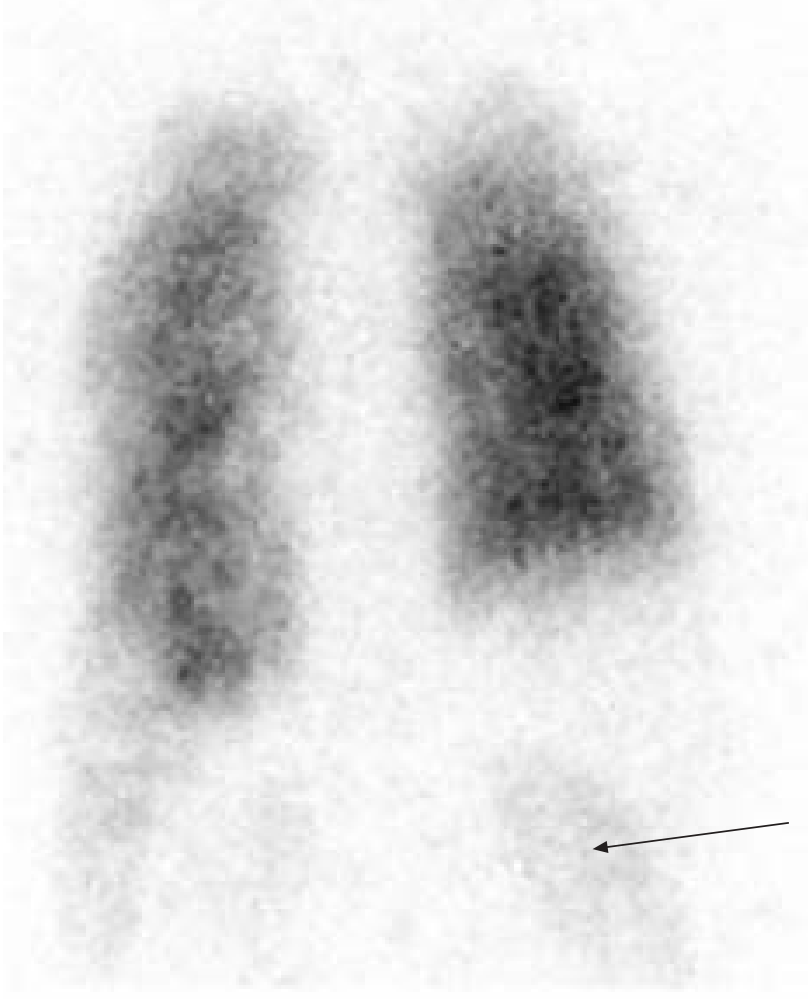

FIGURE 2. Perfusion images from a ventilation-perfusion scan, demonstrating uptake of technetium-99m macro-aggregate albumin in the kidneys (arrow).

As this venous perfusion tracer is usually trapped in the pulmonary vascular bed, evidence of tracer in the kidneys supported the clinical concern for AV shunting. A right-to-left shunt was confirmed by echocardiography (transthoracic and transoesophageal) with saline contrast. Angiography demonstrated a large-volume AV fistula in a tumour in the right
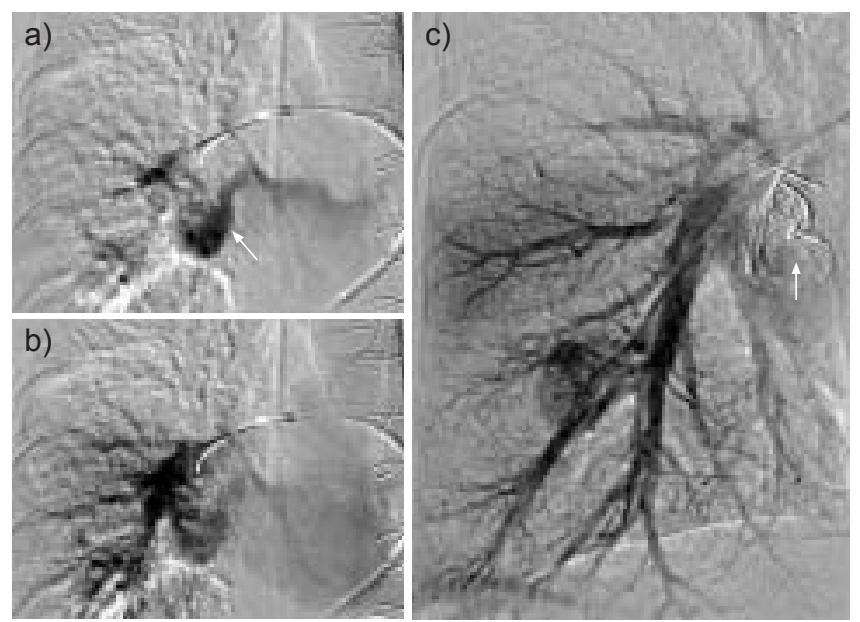

FIGURE 3. Early (a) and delayed (b) imaging from a pulmonary arteriogram, demonstrating shunting into the venous system (arrows). After coiling, shunting is decreased and arterial flow is improved distal to the shunt (c).

middle lobe, from the pulmonary artery to the right superior pulmonary vein. An early image demonstrated rapid shunting into the superior pulmonary vein, whereas a late image showed poor filling of the distal branches of the pulmonary artery (fig. 3a and b). After treatment of the fistula with a coil, good distal perfusion was obtained (fig. 3c), with an immediate improvement in oxygenation. The patient symptomatically improved with a room-air oxygen saturation of $96 \%$. The patient continues to maintain a high functional status and no longer requires supplemental oxygen.

\section{DISCUSSION}

RCCs are known to be highly vascular and often contain small AV fistulas. However, large-vessel, symptomatic AV fistulas are unusual complications of RCC. There have been several reports of high-output heart failure and renovascular hypertension resulting from $\mathrm{AV}$ fistulas in primary renal cell tumours [1]. These complications often resolve after resection. Metastatic sites in the extremities can cause reversal of blood flow and distal ischaemia [2]. AV fistulas from RCC are almost exclusively found in bone [3,4], despite the fact that the lung is a more common site of metastatic disease. This may be due to a microenvironment within bone that is particularly supportive of angiogenesis.

This is the first report of a symptomatic pulmonary arteriovenous fistula from a renal cell carcinoma and only the second report of a symptomatic pulmonary arteriovenous fistula resulting from any tumour [5]. The current study along with previous reports in the literature suggest that the chronicity of tumours plays a role in the development of these fistulas, which are probably due to the dilation of prior subclinical arteriovenous fistulas. Increased vascularity appears to be an intrinsic biological property of renal cell carcinomas. The metabolic rates of renal cell carcinomas do not require the degree of perfusion seen in these tumours. Instead, it appears that activation of the angiogenesis pathway is linked to renal cell tumorigenesis. Von Hippel-Lindau tumour suppressor gene mutations, both sporadic and familial, lead to an 
accumulation of hypoxia-inducible factor- $1 \alpha$, which induces the expression of pro-angiogenic vascular endothelial growth factor. The renal cell carcinoma has a unique susceptibility to anti-angiogenic therapy. This rare complication of renal cell carcinoma reflects its diverse clinical presentations and unique tumour biology.

\section{REFERENCES}

1 Morin RP, Dunn EJ, Wright CB. Renal arteriovenous-fistulas - a review of etiology, diagnosis, and management. Surgery 1986; 99: 114-118.
2 Rodgers MV, Moss AJ, Hoffman M, Lipchik EO. Arteriovenous fistulae secondary to renal cell carcinoma. Clinical and cardiovascular manifestations: report of a case. Circulation 1975; 52: 345-350.

3 Caplan HI, Sparkes RS, Lemay MJ, Mahoney D. Arteriovenous shunt produced by metastatic hypernephroma. JAMA 1961; 177: 788-790.

4 Howlett SA, Caranasos GJ. Metastatic renal cell carcinoma producing arteriovenous shunt. Arch Intern Med 1970; 125: 493-495.

5 Dunn RP, Wexler L. Systemic-to-pulmonary fistula in intrapulmonary Hodgkin's disease. Chest 1974; 66: 590-594. 